\title{
On the upper bound for $\pi_{2}(x)$
}

\author{
by \\ YingChun Cai and MingGao Lu (Shanghai)
}

1. Introduction. For sufficiently large $x$ let

$$
\pi_{2}(x)=\sum_{\substack{p \leq x \\ p+2=p^{\prime}}} 1,
$$

where $p$ and $p^{\prime}$ denote primes. It was showed by Brun [3] in 1919 that

$$
\pi_{2}(x) \leq b \frac{C x}{\log ^{2} x},
$$

for some constant $b>0$, where

$$
C=2 \prod_{p>2}\left(1-\frac{1}{(p-1)^{2}}\right) .
$$

In 1949 Selberg [15] proved that we may take $b=8+o(1)$. In 1964 Pan Chengdong [13] proved that $b=6+o(1)$. In 1962 Wang Yuan [16] pointed out that under the GRH, $b=4+o(1)$, and in 1966 Bombieri and Davenport [1] obtained this result unconditionally.

On the other hand, Hardy and Littlewood [10] conjectured that the asymptotic formula

$$
\pi_{2}(x) \sim \frac{C x}{\log ^{2} x}
$$

should hold, and the arguments in Hua Loo Keng [11] and in Pan Chengdong [14] lead essentially to the same conjecture. Thus the best possible value for $b$ should be $1+o(1)$.

It is rather difficult to reduce the coefficient $b=4+o(1)$. In 1978 Chen Jingrun [4] developed the weighted sieve techniques used in his famous work [5] and proved that

$$
\pi_{2}(x)<\frac{3.9171 C x}{\log ^{2} x} .
$$

2000 Mathematics Subject Classification: 11N36.

Project supported by the National Natural Science Foundation of China (grant no. 10171060, 19801021). 
In recent years, with the development of mean value theorems to large moduli, the coefficient 3.9171 is reduced to

$$
34 / 9 ; \quad 64 / 17 ; \quad 3.5 ; \quad 3.454 ; \quad 3.418
$$

by Fouvry and Iwaniec [8], Fouvry [6], Bombieri, Friedlander and Iwaniec [2], Fouvry and Grupp [7], and Jie Wu [17], respectively. In [17] Chen Jingrun's techniques are applied.

In this paper we shall refine the techniques used in [17] by inserting a new weighted inequality of Chen's type to obtain

THEOREM.

$$
\pi_{2}(x)<\frac{3.406 C x}{\log ^{2} x}
$$

2. $H(s)$ and $G(s)$. In this paper we denote by $\lambda(q)$ a well-factorable function of level $Q$ and of order $k$. For the definition of well-factorable functions we refer the reader to [7].

Lemma 1 ([7]). For an arithmetical function $\lambda^{\prime}$ of level $Q^{\prime}$ and of order $k^{\prime}, Q^{\prime} \leq Q, \lambda \star \lambda^{\prime}$ is a well-factorable function of level $Q Q^{\prime}$ and of order $k+k^{\prime}$.

Let $\mathcal{A}$ denote a finite set of integers, $\mathcal{P}$ an infinite set of primes, and $\overline{\mathcal{P}}$ the set of primes that do not belong to $\mathcal{P}$. For $z \geq 2$, put

$$
\begin{gathered}
P(z)=\prod_{p<z, p \in \mathcal{P}} p, \quad \mathcal{P}(q)=\{p \mid p \in \mathcal{P},(p, q)=1\}, \\
S(\mathcal{A} ; \mathcal{P}, z)=\sum_{a \in \mathcal{A},(a, P(z))=1} 1, \quad \mathcal{A}_{d}=\{a \mid a \in \mathcal{A}, a \equiv 0(\bmod d)\} .
\end{gathered}
$$

Lemma 2 ([12]). Suppose that

$$
\begin{aligned}
\left|\mathcal{A}_{d}\right|= & \frac{\omega(d)}{d} X+r(d), \quad \mu(d) \neq 0, \quad(d, \overline{\mathcal{P}})=1 ; \\
\frac{V\left(z_{1}\right)}{V\left(z_{2}\right)} \leq & \frac{\log z_{2}}{\log z_{1}}\left(1+\frac{K_{1}}{\log z_{1}}\right), \quad K_{1}>1, \quad z_{2}>z_{1} \geq 2 ; \\
& \sum_{\substack{z_{1} \leq p<z_{2} \\
p \in \mathcal{P}}} \sum_{\alpha \geq 2} \frac{\omega\left(p^{\alpha}\right)}{p^{\alpha}} \leq \frac{K_{2}}{\log 3 z_{1}}, \quad K_{2}>1,
\end{aligned}
$$

where $\omega(d)$ is a multiplicative function, $0 \leq \omega(p)<p, X>1$ is independent of $d$, and

$$
V(z)=\prod_{p \mid P(z)}\left(1-\frac{\omega(p)}{p}\right) .
$$


Then for $0<\varepsilon<10^{-5}, 2 \leq z \leq Q^{1 / 2}$, we have

$$
\begin{aligned}
& S(\mathcal{A}, \mathcal{P}, z) \geq X V(z)(f(s)-E)-\sum_{l<L} \sum_{q} \lambda_{l}^{-}(q) r(q), \\
& S(\mathcal{A}, \mathcal{P}, z) \leq X V(z)(F(s)+E)+\sum_{l<L} \sum_{q} \lambda_{l}^{+}(q) r(q),
\end{aligned}
$$

where $\lambda_{l}^{ \pm}$are well-factorable functions of level $Q$ and

$$
\begin{gathered}
L=\exp \left(8 \varepsilon^{-3}\right), \quad E \ll \varepsilon+\varepsilon^{-8} \exp \left(K_{1}+K_{2}\right) \log ^{-1 / 3} Q, \\
s=\frac{\log Q}{\log z}, \quad\left|\lambda_{l}^{ \pm}(q)\right| \leq 1, \quad \lambda_{l}^{ \pm}(q)=0 \quad \text { for }(q, P(z))=1 .
\end{gathered}
$$

$f(s)$ and $F(s)$ are determined by the following differential-difference equation:

$$
\left\{\begin{array}{l}
F(s)=2 e^{\gamma} / s, \quad f(s)=0, \quad 0<s \leq 2, \\
(s F(s))^{\prime}=f(s-1), \quad(s f(s))^{\prime}=F(s-1), \quad s \geq 2 ;
\end{array}\right.
$$

here and below, $\gamma$ is Euler's constant.

Lemma 3 ([9]). We have

$$
\begin{aligned}
& F(s)= \begin{cases}2 e^{\gamma} / s, & 0<s \leq 3, \\
\frac{2 e^{\gamma}}{s}\left(1+\int_{2}^{s-1} \frac{\log (t-1)}{t} d t\right), & 3 \leq s \leq 5 ;\end{cases} \\
& f(s)=\frac{2 e^{\gamma} \log (s-1)}{s}, \quad 2 \leq s \leq 4 .
\end{aligned}
$$

Lemma 4 ([2]). Let $Q=x^{4 / 7-\varepsilon}, \varepsilon>0$. For any given $A>0$ and $|a| \leq$ $\log ^{A} x$,

$$
\sum_{(q, a)=1} \lambda(q)\left(\pi(x ; q, a)-\frac{\operatorname{Li} x}{\varphi(q)}\right)=O_{A, \varepsilon, a}\left(\frac{x}{\log ^{A} x}\right) .
$$

Throughout this paper we shall take

$$
\mathcal{A}=\{p+2 \mid p \leq x\}, \quad \mathcal{P}=\{p \mid p>2\} .
$$

Then

$$
V(z)=C \frac{e^{-\gamma}}{\log z}\left(1+O\left(\frac{1}{\log z}\right)\right) .
$$

For the definitions of $H(s)$ and $G(s)$ we refer the reader to [17], but for the sake of completeness we repeat them here.

For $\varepsilon>0$ let

$$
Q=x^{4 / 7-\varepsilon}, \quad W=x^{\varepsilon} .
$$

By $\Pi_{[Y, Z)}$ we denote the characteristic function of the set of the primes in the interval $[Y, Z)$. Let $\mathcal{U}_{0}$ denote the set which consists of the characteristic 
function $\chi$ of the set $\{1\}$. For $k=1,2, \ldots$, we denote by $\mathcal{U}_{k}$ the set of arithmetical functions $\chi$ of the form

$$
\chi=\Pi_{\left[V_{1}, \Delta V_{1}\right)} \star \ldots \star \Pi_{\left[V_{i}, \Delta V_{i}\right)}
$$

with

$$
\begin{aligned}
& 1 \leq i \leq k, \quad 1+\log ^{-4} x \leq \Delta \leq 1+2 \log ^{-4} x, \\
& V_{1} \geq \ldots \geq V_{i} \geq W \\
& V_{1}^{2} \leq Q, \quad V_{1} V_{2}^{2} \leq Q, \ldots, \quad V_{1} \ldots V_{i-1} V_{i}^{2} \leq Q
\end{aligned}
$$

By Lemma 1 of [12] we know that for any $\chi \in \mathcal{U}_{k}, \chi$ is a well-factorable function of level $Q$ and of order $k$.

For any $q \leq Q W^{-1}$ put $\underline{q}=Q / q$. For any $\chi \in \mathcal{U}_{k}$ we have

$$
\Xi(\chi ; x)=\sum \frac{2 \chi(q) C x}{\varphi(q) \log x \log \underline{q}}>\frac{2 C x}{4^{k} \log ^{5 k+2} x} .
$$

By Lemmas 1,2 and some routine arguments we get

$$
\begin{aligned}
& \sum \chi(q) S\left(\mathcal{A}_{q}, \mathcal{P}(q), \underline{q}^{1 / s}\right) \leq \Xi(\chi ; x)(A(s)+o(1)), \\
& \sum \chi(q) S\left(\mathcal{A}_{q}, \mathcal{P}(q), \underline{q}^{1 / s}\right) \geq \Xi(\chi ; x)(B(s)+o(1)),
\end{aligned}
$$

where

$$
A(s)=\frac{s F(s)}{2 e^{\gamma}}, \quad B(s)=\frac{s f(s)}{2 e^{\gamma}} .
$$

In view of (2.2) and (2.3) it is reasonable to define $H_{x, \chi}(s)$ and $G_{x, \chi}(s)$ as the supremum of $h>-\infty$ such that

$$
\begin{aligned}
& \sum \chi(q) S\left(\mathcal{A}_{q}, \mathcal{P}(q), \underline{q}^{1 / s}\right) \leq \Xi(\chi ; x)(A(s)-h), \\
& \sum \chi(q) S\left(\mathcal{A}_{q}, \mathcal{P}(q), \underline{q}^{1 / s}\right) \geq \Xi(\chi ; x)(B(s)+h),
\end{aligned}
$$

respectively. Let

$$
H(s)=\sup _{x>x_{0}} \sup _{k \geq 0} \sup _{\chi \in \mathcal{U}_{k}} H_{x, \chi}(s), \quad G(s)=\sup _{x>x_{0}} \sup _{k \geq 0} \sup _{\chi \in \mathcal{U}_{k}} G_{x, \chi}(s) .
$$

Then we have

Proposition 1 ([17]). (1) $H(s)$ is decreasing for $1 \leq s \leq 3$.

(2) We have the inequalities

$$
\begin{aligned}
& \sum \chi(q) S\left(\mathcal{A}_{q}, \mathcal{P}(q), \underline{q}^{1 / s}\right) \leq \Xi(\chi ; x)(A(s)-H(s)), \\
& \sum \chi(q) S\left(\mathcal{A}_{q}, \mathcal{P}(q), \underline{q}^{1 / s}\right) \geq \Xi(\chi ; x)(B(s)+G(s)) .
\end{aligned}
$$

(3) $0 \leq H(s) \leq 1,0 \leq G(s) \leq 1$. 
In what follows, we shall denote by $\chi$ a well-factorable function of level $Q$ and of order $k$ such that $\chi \in \mathcal{U}_{k}$ and

$$
\Xi(\chi ; x)=\sum \frac{2 \chi(q) C x}{\varphi(q) \log x \log \underline{q}}>\frac{2 C x}{4^{k} \log ^{5 k+2} x} .
$$

Proposition 2 ([17]). For $2 \leq s \leq s^{\prime} \leq 10$ we have

$$
H(s) \geq H\left(s^{\prime}\right)+\int_{s-1}^{s^{\prime}-1} \frac{G(t)}{t} d t-\varepsilon, \quad G(s) \geq G\left(s^{\prime}\right)+\int_{s-1}^{s^{\prime}-1} \frac{H(t)}{t} d t-\varepsilon .
$$

Proposition 3. We have

$$
\begin{aligned}
G(4) \geq & 0.02238 H(2.2)+0.00443 H(2.3)+0.00479 H(2.4) \\
& +0.00515 H(2.5)+0.00550 H(2.6)+0.00583 H(2.7) \\
& +0.00615 H(2.8)+0.00645 H(2.9)+0.00676 H(3)-\varepsilon .
\end{aligned}
$$

Proof. By Propositions 1, 2 we have

$$
\begin{aligned}
& H(s) \geq H(5)+\int_{s-1}^{4} \frac{G(t)}{t} d t-\varepsilon \geq \int_{s-1}^{4} \frac{G(t)}{t} d t-\varepsilon \\
& G(4) \geq G(6)+\int_{3}^{5} \frac{H(t)}{t} d t-\varepsilon \geq \int_{3}^{5} \frac{H(t)}{t} d t-\varepsilon \\
& G(s) \geq G(4)+\int_{s-1}^{3} \frac{H(t)}{t} d t-\varepsilon, \\
& G(4) \geq \int_{3}^{5} \frac{H(t)}{t} d t-\varepsilon \geq \int_{3}^{5} \frac{d t}{t} \int_{t-1}^{4} \frac{G(s)}{s} d s-\varepsilon \\
& \geq \int_{3}^{5} \frac{d t}{t} \int_{t-1}^{4} \frac{1}{s}\left(G(4)+\int_{s-1}^{3} \frac{H(u)}{u} d u\right) d s-\varepsilon \\
&= G(4) \int_{3}^{5} \frac{\log \frac{4}{t-1}}{t} d t+\int_{1}^{3} \frac{H(u)}{u} d u \int_{3}^{u+2} \frac{\log \frac{u+1}{t-1}}{t} d t-\varepsilon \\
& \geq 0.17168 G(4)+0.01854 H(2.2)+0.00367 H(2.3) \\
&+0.00397 H(2.4)+0.00427 H(2.5)+0.00456 H(2.6) \\
&+0.00483 H(2.7)+0.00510 H(2.8)+0.00535 H(2.9) \\
&+0.00560 H(3)-\varepsilon,
\end{aligned}
$$

and Proposition 3 follows easily. 
Lemma $5([17])$. Let $x>1, z=x^{1 / u}, Q(z)=\prod_{p<z} p$. Then

$$
\sum_{\substack{n \leq x \\(n, Q(z))=1}} 1=w(u) \frac{x}{\log z}+O\left(\frac{x}{\log ^{2} z}\right),
$$

where $w(u)$ is determined by the following differential-difference equation:

$$
\left\{\begin{array}{l}
w(u)=0, \quad 0<u<1 \\
w(u)=1 / u, \quad 1 \leq u \leq 2 \\
(u w(u))^{\prime}=w(u-1), \quad u \geq 2 .
\end{array}\right.
$$

Moreover,

$$
w(u)<\frac{1}{1.763} \quad \text { for } u \geq 1.763 .
$$

Lemma 6 ([17]). For any $\chi \in \mathcal{U}_{k}, k \geq 0$ and $x^{1 / 2} \leq q \chi(q) M, 2 q \chi(q) M$ $\leq x, M<M^{\prime} \leq 2 M$, we have

$$
\begin{aligned}
& \sum \chi(q) \sum_{M \leq m \leq M^{\prime}} a(m) S\left(\mathcal{A}_{q m}, \mathcal{P}(q m), z(m)\right) \\
& \leq(1+o(1)) \frac{3.5 C}{\log x} \sum \chi(q) \sum_{M \leq m \leq M^{\prime}} a(m) \sum_{\substack{n \leq x /(q m) \\
(n, Q(z(m)))=1}} 1+O\left(\frac{x}{\log ^{5 k+100} x}\right),
\end{aligned}
$$

where $0 \leq a(m) \ll 1$ for $M \leq m \leq M^{\prime}$.

Lemma 7 ([17]). For $2.5 \leq u<3<v<4$ we have

$$
\begin{aligned}
H(u) \geq & H(v)-\int_{2}^{v-1} \frac{\log (t-1)}{t} d t \\
& +\int_{u-1}^{v-1} \frac{\log \left(v-1-\frac{v}{t+1}\right)}{2 t} d t+\int_{u-1}^{v-1} \frac{G\left(v-\frac{v}{t+1}\right)}{2 t} d t \\
& -\frac{7}{8 \cdot 1.763}\left((u+v) \log \frac{v}{u}-2(v-u)\right)-\varepsilon .
\end{aligned}
$$

Lemma 8. We have

$$
\begin{aligned}
H(2.5) \geq & 0.013948+0.15751 H(2.2)+0.02478 H(2.3) \\
& +0.02544 H(2.4)+0.02653 H(2.6)+0.02699 H(2.7) \\
& +0.02739 H(2.8)+0.02774 H(2.9)+0.02806 H(3) ; \\
H(2.6) \geq & 0.01091+0.15796 H(2.2)+0.02420 H(2.3) \\
& +0.02487 H(2.4)+0.02547 H(2.5)+0.02647 H(2.7) \\
& +0.02688 H(2.8)+0.02724 H(2.9)+0.02757 H(3) ;
\end{aligned}
$$




$$
\begin{array}{rl}
H(2.7) \geq 0 & 0.00733+0.16626 H(2.2)+0.02388 H(2.3) \\
& +0.02455 H(2.4)+0.02516 H(2.5)+0.02570 H(2.6) \\
& +0.02659 H(2.8)+0.02692 H(2.9)+0.02729 H(3) ; \\
H(2.8) \geq & 0.00383+0.1675 H(2.2)+0.02389 H(2.3) \\
& +0.02457 H(2.4)+0.02518 H(2.5)+0.02572 H(2.6) \\
& +0.02620 H(2.7)+0.02699 H(2.9)+0.02732 H(3) ; \\
H(2.9) \geq 0 & 0.0011+0.17847 H(2.2)+0.02432 H(2.3) \\
& +0.02498 H(2.4)+0.02559 H(2.5)+0.02612 H(2.6) \\
& +0.02658 H(2.7)+0.02699 H(2.8)+0.02768 H(3) .
\end{array}
$$

Proof. By (2.4), (2.5) we have

$$
\begin{aligned}
& H(v) \geq \int_{v-1}^{4} \frac{G(t)}{t} d t-\varepsilon \geq \int_{v-1}^{4}\left(G(4)+\int_{s-1}^{3} \frac{H(s)}{s} d s\right) \frac{d t}{t}-\varepsilon \\
&=G(4) \log \frac{4}{v-1}+\int_{v-2}^{3} \frac{H(s)}{s} \log \frac{s+1}{v-1} d s-\varepsilon \\
& \int_{u-1}^{v-1} \frac{G\left(v-\frac{v}{t+1}\right)}{t} d t=v \int_{v-v / u}^{v-1} \frac{G(s)}{s(v-s)} d s \\
& \geq \int_{v-v / u}^{v-1}\left(G(4)+\int_{s-1}^{3} \frac{H(t)}{t} d t\right) \frac{v}{s(v-s)} d s-\varepsilon \\
&=G(4) \log \frac{v-1}{u-1}+\int_{v-2}^{3} \frac{H(t)}{t} \log \frac{v-1}{u-1} d t \\
&+\int_{v-v / u-1}^{v-2} \frac{H(t)}{t} \log \frac{t+1}{(u-1)(v-1-t)} d t-\varepsilon .
\end{aligned}
$$

By (2.6), (2.7) and Lemma 7 we get

$$
\begin{aligned}
H(u) \geq & J(u, v)+\frac{G(4)}{2} \log \frac{16}{(v-1)(u-1)} \\
& +\int_{v-v / u-1}^{v-2} \frac{H(t)}{2 t} \log \frac{t+1}{(u-1)(v-1-t)} d t \\
& +\int_{v-2}^{3} \frac{H(t)}{2 t} \log \frac{(t+1)^{2}}{(v-1)(u-1)} d t,
\end{aligned}
$$

where 


$$
\begin{aligned}
J(u, v)= & \int_{u-1}^{v-1} \frac{\log \left(v-1-\frac{v}{t+1}\right)}{2 t} d t-\int_{2}^{v-1} \frac{\log (t-1)}{t} d t \\
& -\frac{7}{8 \cdot 1.763}\left((u+v) \log \frac{v}{u}-2(v-u)\right)-\varepsilon .
\end{aligned}
$$

By (2.8) with

$$
\begin{array}{ll}
(u, v)=(2.5,3.72), & (u, v)=(2.6,3.61), \\
(u, v)=(2.7,3.49), & (u, v)=(2.8,3.35), \\
(u, v)=(2.9,3.19) &
\end{array}
$$

and some computation we get the assertion of Lemma 8 .

LEMma 9. We have

$$
\begin{aligned}
H(3) \geq & 0.19649 H(2.2)+0.02531 H(2.3)+0.02598 H(2.4) \\
& +0.02654 H(2.5)+0.02705 H(2.6)+0.02749 H(2.7) \\
& +0.02788 H(2.8)+0.02822 H(2.9)-\varepsilon .
\end{aligned}
$$

Proof. By (2.6) and Proposition 3 we have

$$
\begin{aligned}
\text { (2.9) } H(3) \geq & G(4) \log 2+\int_{1}^{3} \frac{H(s)}{s} \log \frac{s+1}{2} d s-\varepsilon \\
\geq & 0.19104 H(2.2)+0.02461 H(2.3)+0.02526 H(2.4) \\
& +0.02581 H(2.5)+0.02630 H(2.6)+0.02673 H(2.7) \\
& +0.02711 H(2.8)+0.02744 H(2.9)+0.02774 H(3)-\varepsilon .
\end{aligned}
$$

By (2.9) we get Lemma 9 easily.

Lemma 10 ([17]). For $2<u \leq 2.5<3<v<5$ we have

$$
\begin{aligned}
H(u) \geq & H(v)+K(u, v)+\int_{u-1}^{v-1} \frac{G\left(v-1-\frac{v}{t+1}\right)}{t} d t \\
& +\int_{2}^{v-1} \frac{G\left(v-1-\frac{v}{t+1}\right)}{t} d t \\
& +H(2.2) \int_{2}^{v-1} \frac{\log \left(v-1-\frac{v}{t+1}\right)-\log (t-1)}{3 t} d t-\varepsilon,
\end{aligned}
$$

where

$$
\begin{aligned}
K(u, v)= & \int_{u-1}^{v-1} \frac{\log \left(v-1-\frac{v}{t+1}\right)}{3 t} d t-2 \int_{2}^{v-1} \frac{\log (t-1)}{3 t} d t \\
& -\frac{7}{12 \cdot 1.763}\left(v-3-3 \log \frac{v}{3}\right) \log \frac{3}{u}
\end{aligned}
$$




$$
\begin{aligned}
& -\frac{7}{12 \cdot 1.763}\left(u-3+3 \log \frac{3}{u}\right) \log \frac{v}{3} \\
& -\frac{7}{24}\left(u \log ^{2} \frac{3}{u}+(6+4 u) \log \frac{3}{u}+6 u-18\right) .
\end{aligned}
$$

REMARK. It should be pointed out that there is a mistake in Lemme 11 of [17] where the term

is missing.

$$
\int_{2}^{v-1} \frac{G\left(v-1-\frac{v}{t+1}\right)}{t} d t
$$

Lemma 11. We have

$$
\begin{aligned}
H(2.3) \geq & 0.01770+0.14833 H(2.2)+0.02567 H(2.4) \\
& +0.02624 H(2.5)+0.02675 H(2.6)+0.02720 H(2.7) \\
& +0.02759 H(2.8)+0.02793 H(2.9)+0.02825 H(3) ; \\
H(2.4) \geq & 0.01559+0.14941 H(2.2)+0.02444 H(2.3) \\
& +0.02570 H(2.5)+0.02622 H(2.6)+0.02668 H(2.7) \\
& +0.02709 H(2.8)+0.02744 H(2.9)+0.02777 H(3) .
\end{aligned}
$$

Proof. By (2.6), (2.7) and Lemma 10 we get

$$
\begin{aligned}
H(u) \geq & K(u, v)+\frac{G(4)}{3} \log \frac{32}{(v-1)(u-1)} \\
& +\int_{v-v / u-1}^{2 v / 3-1} \frac{H(t)}{3 t} \log \frac{t+1}{(u-1)(v-1-t)} d t \\
& +\int_{2 v / 3-1}^{v-2} \frac{H(t)}{3 t} \log \frac{(t+1)^{2}}{2(u-1)(v-1-t)^{2}} d t \\
& +\int_{v-2}^{3} \frac{H(t)}{3 t} \log \frac{(t+1)^{3}}{2(v-1)(u-1)} d t \\
& +H(2.2) \int_{2}^{v-1} \frac{\log \left(v-1-\frac{v}{t+1}\right)-\log (t-1)}{3 t} d t-\varepsilon .
\end{aligned}
$$

By $(2.10)$ with $(u, v)=(2.3,4.12),(u, v)=(2.4,4)$ and some computation we get the assertion.

3. Proof of the Theorem. By Buchstab's identity, we have

$$
S\left(\mathcal{A}_{q}, \mathcal{P}(q), \underline{q}^{1 / 2.2}\right)=S\left(\mathcal{A}_{q}, \mathcal{P}(q), \underline{q}^{1 / 4.4}\right)-\sum_{\underline{q}^{1 / 4.4} \leq p<\underline{q}^{1 / 2.2}} S\left(\mathcal{A}_{p q}, \mathcal{P}(q), p\right),
$$


(3.2)

$$
\begin{aligned}
& S\left(\mathcal{A}_{q}, \mathcal{P}(q), \underline{q}^{1 / 2.2}\right) \\
= & S\left(\mathcal{A}_{q}, \mathcal{P}(q), \underline{q}^{1 / 4.4}\right)-\sum_{\underline{q}^{1 / 4.4} \leq p<\underline{q}^{1 / 3}} S\left(\mathcal{A}_{p q}, \mathcal{P}(q), \underline{q}^{1 / 4.4}\right) \\
& +\sum_{\underline{q}^{1 / 4.4} \leq p_{1}<p_{2}<\underline{q}^{1 / 3}} S\left(\mathcal{A}_{p_{1} p_{2} q}, \mathcal{P}(q), p_{1}\right)-\sum_{\underline{q}^{1 / 3} \leq p<\underline{q}^{1 / 2.2}} S\left(\mathcal{A}_{p q}, \mathcal{P}(q), p\right),
\end{aligned}
$$

(3.3) $S\left(\mathcal{A}_{q}, \mathcal{P}(q), \underline{q}^{1 / 2.2}\right)$

$$
=S\left(\mathcal{A}_{q}, \mathcal{P}(q), \underline{q}^{1 / 3.6}\right)-\sum_{\underline{q}^{1 / 3.6} \leq p<\underline{q}^{1 / 2.2}} S\left(\mathcal{A}_{p q}, \mathcal{P}(q), p\right) .
$$

By (3.1)-(3.3) we have

$$
\begin{aligned}
& 3 S\left(\mathcal{A}_{q}, \mathcal{P}(q), \underline{q}^{1 / 2.2}\right) \\
= & 2 S\left(\mathcal{A}_{q}, \mathcal{P}(q), \underline{q}^{1 / 4.4}\right)+S\left(\mathcal{A}_{q}, \mathcal{P}(q), \underline{q}^{1 / 3.6}\right) \\
- & \sum_{\underline{q}^{1 / 4.4} \leq p<\underline{q}^{1 / 3}} S\left(\mathcal{A}_{p q}, \mathcal{P}(q), \underline{q}^{1 / 4.4}\right)-\sum_{\underline{q}^{1 / 4.4} \leq p<\underline{q}^{1 / 2.2}} S\left(\mathcal{A}_{p q}, \mathcal{P}(q), p\right) \\
- & \sum_{\underline{q}^{1 / 3.6} \leq p<\underline{q}^{1 / 2.2}} S\left(\mathcal{A}_{p q}, \mathcal{P}(q), p\right)+\sum_{\underline{q}^{1 / 4.4} \leq p_{1}<p_{2}<\underline{q}^{1 / 3}} S\left(\mathcal{A}_{p_{1} p_{2} q}, \mathcal{P}(q), p_{1}\right) \\
- & \sum_{\underline{q}^{1 / 3} \leq p<\underline{q}^{1 / 2.2}} S\left(\mathcal{A}_{p q}, \mathcal{P}(q), p\right) .
\end{aligned}
$$

By Buchstab's identity, we have

$$
\begin{aligned}
S\left(\mathcal{A}_{q}, \mathcal{P}(q), \underline{q}^{1 / 2.2}\right) & \\
= & S\left(\mathcal{A}_{q}, \mathcal{P}(q), \underline{q}^{1 / 4.5}\right)-\frac{1}{2} \sum_{\underline{q}^{1 / 4.5} \leq p<\underline{q}^{1 / 4.4}} S\left(\mathcal{A}_{p q}, \mathcal{P}(q), p\right) \\
& -\frac{1}{2} \sum_{\underline{q}^{1 / 4.5} \leq p<\underline{q}^{1 / 4.4}} S\left(\mathcal{A}_{p q}, \mathcal{P}(q), \underline{q}^{1 / 4.5}\right) \\
& +\frac{1}{2} \sum_{\underline{q}^{1 / 4.5} \leq p_{1}<p_{2}<\underline{q}^{1 / 4.4}} S\left(\mathcal{A}_{p_{1} p_{2} q}, \mathcal{P}(q), p_{1}\right) \\
& -\sum_{\underline{q}^{1 / 4.4} \leq p<\underline{q}^{1 / 3}} S\left(\mathcal{A}_{p q}, \mathcal{P}(q), \underline{q}^{1 / 4.4}\right) \\
& +\sum_{\underline{q}^{1 / 4.4} \leq p_{1}<p_{2}<\underline{q}^{1 / 3.6}} S\left(\mathcal{A}_{p_{1} p_{2} q}, \mathcal{P}(q), p_{1}\right)
\end{aligned}
$$




$$
\begin{aligned}
& +\sum_{\underline{q}^{1 / 4.4} \leq p_{1}<\underline{q}^{1 / 3.6} \leq p_{2}<\underline{q}^{1 / 3}} S\left(\mathcal{A}_{p_{1} p_{2} q}, \mathcal{P}(q), p_{1}\right) \\
& +\sum_{\underline{q}^{1 / 3.6} \leq p_{1}<p_{2}<\underline{q}^{1 / 3}} S\left(\mathcal{A}_{p_{1} p_{2} q}, \mathcal{P}(q), p_{1}\right) \\
& -\sum_{\underline{q}^{1 / 3} \leq p<\underline{q}^{1 / 2.4}} S\left(\mathcal{A}_{p q}, \mathcal{P}(q), \underline{q}^{1 / 4.5}\right) \\
& +\sum_{\underline{q}^{1 / 4.5} \leq p_{1}<\underline{q}^{1 / 4.4}<\underline{q}^{1 / 3} \leq p_{2}<\underline{q}^{1 / 2.4}} S\left(\mathcal{A}_{p_{1} p_{2} q}, \mathcal{P}(q), p_{1}\right) \\
& +\sum_{\underline{q}^{1 / 4.4} \leq p_{1}<\underline{q}^{1 / 3.6}<\underline{q}^{1 / 3} \leq p_{2}<\underline{q}^{1 / 2.4}} S\left(\mathcal{A}_{p_{1} p_{2} q}, \mathcal{P}(q), \underline{q}^{1 / 4.5}\right) \\
& \sum_{\underline{q}^{1 / 4.5} \leq p_{1}<\underline{q}^{1 / 4.4} \leq p_{2}<\underline{q}^{1 / 3.6}<\underline{q}^{1 / 3} \leq p_{3}<\underline{q}^{1 / 2.4}} S\left(\mathcal{A}_{p_{1} p_{2} p_{3} q}, \mathcal{P}(q), p_{1}\right) \\
& \sum_{\underline{q}^{1 / 4.4} \leq p_{1}<p_{2}<\underline{q}^{1 / 3.6}<\underline{q}^{1 / 3} \leq p_{3}<\underline{q}^{1 / 2.4}} S\left(\mathcal{A}_{p_{1} p_{2} p_{3} q}, \mathcal{P}(q), p_{1}\right) \\
& +\sum_{\underline{q}^{1 / 3.6} \leq p_{1}<\underline{q}^{1 / 3} \leq p_{2}<\underline{q}^{1 / 2.4}} S\left(\mathcal{A}_{p_{1} p_{2} q}, \mathcal{P}(q), p_{1}\right) \\
& +\sum_{\underline{q}^{1 / 3} \leq p_{1}<p_{2}<\underline{q}^{1 / 2.4}} S\left(\mathcal{A}_{p_{1} p_{2} q}, \mathcal{P}(q), p_{1}\right) \\
& -\sum_{\underline{q}^{1 / 2.4} \leq p<\underline{q}^{1 / 2.2}} S\left(\mathcal{A}_{p q}, \mathcal{P}(q), p\right),
\end{aligned}
$$

and

(3.6) $S\left(\mathcal{A}_{q}, \mathcal{P}(q), \underline{q}^{1 / 2.2}\right)$

$$
\begin{aligned}
= & S\left(\mathcal{A}_{q}, \mathcal{P}(q), \underline{q}^{1 / 4.5}\right)-\sum_{\underline{q}^{1 / 4.5} \leq p<\underline{q}^{1 / 2.2}} S\left(\mathcal{A}_{p q}, \mathcal{P}(q), \underline{q}^{1 / 4.5}\right) \\
& +\frac{1}{2} \sum_{\underline{q}^{1 / 4.5} \leq p_{1}<p_{2}<\underline{q}^{1 / 4.4}} S\left(\mathcal{A}_{p_{1} p_{2} q}, \mathcal{P}(q), p_{1}\right) \\
& +\frac{1}{2} \sum_{\underline{q}^{1 / 4.5} \leq p_{1}<p_{2}<\underline{q}^{1 / 4.4}} S\left(\mathcal{A}_{p_{1} p_{2} q}, \mathcal{P}(q), \underline{q}^{1 / 4.5}\right) \\
& -\frac{1}{2} \sum_{\underline{q}^{1 / 4.5} \leq p_{1}<p_{2}<p_{3}<\underline{q}^{1 / 4.4}} S\left(\mathcal{A}_{p_{1} p_{2} p_{3} q}, \mathcal{P}(q), p_{1}\right)
\end{aligned}
$$




$$
\begin{aligned}
& +\frac{1}{2} \sum_{\underline{q}^{1 / 4.5} \leq p_{1}<\underline{q}^{1 / 4.4} \leq p_{2}<\underline{q}^{1 / 2.2}} S\left(\mathcal{A}_{p_{1} p_{2} q}, \mathcal{P}(q), p_{1}\right) \\
& +\frac{1}{2} \sum_{\underline{q}^{1 / 4.5} \leq p_{1}<\underline{q}^{1 / 4.4} \leq p_{2}<\underline{q}^{1 / 2.2}} S\left(\mathcal{A}_{p_{1} p_{2} q}, \mathcal{P}(q), \underline{q}^{1 / 4.5}\right) \\
& -\frac{1}{2} \sum_{\underline{q}^{1 / 4.5} \leq p_{1}<p_{2}<\underline{q}^{1 / 4.4} \leq p_{3}<\underline{q}^{1 / 2.2}} S\left(\mathcal{A}_{p_{1} p_{2} p_{3} q}, \mathcal{P}(q), p_{1}\right) \\
& +\sum_{\underline{q}^{1 / 4.4} \leq p_{1}<p_{2}<\underline{q}^{1 / 3}} S\left(\mathcal{A}_{p_{1} p_{2} q}, \mathcal{P}(q), \underline{q}^{1 / 4.5}\right) \\
& -\sum_{\underline{q}^{1 / 4.5} \leq p_{1}<\underline{q}^{1 / 4.4} \leq p_{2}<p_{3}<\underline{q}^{1 / 3}} S\left(\mathcal{A}_{p_{1} p_{2} p_{3} q}, \mathcal{P}(q), p_{1}\right) \\
& -\sum_{\underline{q}^{1 / 4.4} \leq p_{1}<p_{2}<p_{3}<\underline{q}^{1 / 3}} S\left(\mathcal{A}_{p_{1} p_{2} p_{3} q}, \mathcal{P}(q), p_{1}\right) \\
& +\sum_{\underline{q}^{1 / 4.4} \leq p_{1}<\underline{q}^{1 / 3} \leq p_{2}<\underline{q}^{1 / 2.2}} S\left(\mathcal{A}_{p_{1} p_{2} q}, \mathcal{P}(q), p_{1}\right) \\
& +\sum_{\underline{q}^{1 / 3} \leq p_{1}<p_{2}<\underline{q}^{1 / 2.2}} S\left(\mathcal{A}_{p_{1} p_{2} q}, \mathcal{P}(q), p_{1}\right) .
\end{aligned}
$$

By (3.4)-(3.6) we get

$$
5 \sum \chi(q) S\left(\mathcal{A}_{q}, \mathcal{P}(q), \underline{q}^{1 / 2.2}\right)=\sum \chi(q)\left(S_{q}^{1}+S_{q}^{2}+S_{q}^{3}\right),
$$

where

$$
\begin{aligned}
S_{q}^{1}= & 2 S\left(\mathcal{A}_{q}, \mathcal{P}(q), \underline{q}^{1 / 4.5}\right)+2 S\left(\mathcal{A}_{q}, \mathcal{P}(q), \underline{q}^{1 / 4.4}\right) \\
& +S\left(\mathcal{A}_{q}, \mathcal{P}(q), \underline{q}^{1 / 3.6}\right)-2 \sum_{\underline{q}^{1 / 4.4} \leq p<\underline{q}^{1 / 3}} S\left(\mathcal{A}_{p q}, \mathcal{P}(q), \underline{q}^{1 / 4.4}\right) \\
& -\frac{1}{2} \sum_{\underline{q}^{1 / 4.5} \leq p<\underline{q}^{1 / 4.4}} S\left(\mathcal{A}_{p q}, \mathcal{P}(q), \underline{q}^{1 / 4.5}\right) \\
& -\sum_{\underline{q}^{1 / 3} \leq p<\underline{q}^{1 / 2.4}} S\left(\mathcal{A}_{p q}, \mathcal{P}(q), \underline{q}^{1 / 4.5}\right) \\
& -\sum_{\underline{q}^{1 / 4.5} \leq p<\underline{q}^{1 / 2.2}} S\left(\mathcal{A}_{p q}, \mathcal{P}(q), \underline{q}^{1 / 4.5}\right) \\
+ & \frac{1}{2} \sum_{\underline{q}^{1 / 4.5} \leq p_{1}<p_{2}<\underline{q}^{1 / 4.4}} S\left(\mathcal{A}_{p_{1} p_{2} q}, \mathcal{P}(q), \underline{q}^{1 / 4.5}\right)
\end{aligned}
$$




$$
\begin{aligned}
& +\frac{1}{2} \sum_{\underline{q}^{1 / 4.5} \leq p_{1}<p_{2}<\underline{q}^{1 / 4.4}} S\left(\mathcal{A}_{p_{1} p_{2} q}, \mathcal{P}(q), p_{1}\right) \\
& +\sum_{\underline{q}^{1 / 4.4} \leq p_{1}<p_{2}<\underline{q}^{1 / 3}} S\left(\mathcal{A}_{p_{1} p_{2} q}, \mathcal{P}(q), \underline{q}^{1 / 4.5}\right) \\
& +\frac{1}{2} \sum_{\underline{q}^{1 / 4.5} \leq p_{1}<\underline{q}^{1 / 4.4} \leq p_{2}<\underline{q}^{1 / 2.2}} S\left(\mathcal{A}_{p_{1} p_{2} q}, \mathcal{P}(q), \underline{q}^{1 / 4.5}\right) \\
& +\sum_{\underline{q}^{1 / 4.4} \leq p_{1}<p_{2}<\underline{q}^{1 / 3.6}} S\left(\mathcal{A}_{p_{1} p_{2} q}, \mathcal{P}(q), p_{1}\right) \\
& +\sum_{\underline{q}^{1 / 4.4} \leq p_{1}<\underline{q}^{1 / 3.6} \leq p_{2}<\underline{q}^{1 / 3}} S\left(\mathcal{A}_{p_{1} p_{2} q}, \mathcal{P}(q), p_{1}\right) \\
& +\sum_{\underline{q}^{1 / 4.5} \leq p_{1}<\underline{q}^{1 / 4.4}<\underline{q}^{1 / 3} \leq p_{2}<\underline{q}^{1 / 2.4}} S\left(\mathcal{A}_{p_{1} p_{2} q}, \mathcal{P}(q), p_{1}\right) \\
& +\sum_{\underline{q}^{1 / 4.4} \leq p_{1}<\underline{q}^{1 / 3.6}<\underline{q}^{1 / 3} \leq p_{2}<\underline{q}^{1 / 2.4}} S\left(\mathcal{A}_{p_{1} p_{2} q}, \mathcal{P}(q), \underline{q}^{1 / 4.5}\right), \\
& \text { (3.9) } S_{q}^{2}=\sum_{\underline{q}^{1 / 4.4} \leq p_{1}<p_{2}<\underline{q}^{1 / 2.2}} S\left(\mathcal{A}_{p_{1} p_{2} q}, \mathcal{P}(q), p_{1}\right) \\
& +\sum_{\underline{q}^{1 / 3.6} \leq p_{1}<p_{2}<\underline{q}^{1 / 2.4}} S\left(\mathcal{A}_{p_{1} p_{2} q}, \mathcal{P}(q), p_{1}\right) \\
& -\sum_{\underline{q}^{1 / 4.4} \leq p<\underline{q}^{1 / 2.2}} S\left(\mathcal{A}_{p q}, \mathcal{P}(q), p\right) \\
& -\sum_{\underline{q}^{1 / 3.6} \leq p<\underline{q}^{1 / 2.2}} S\left(\mathcal{A}_{p q}, \mathcal{P}(q), p\right) \\
& -\sum_{\underline{q}^{1 / 4.4} \leq p_{1}<p_{2}<p_{3}<\underline{q}^{1 / 3}} S\left(\mathcal{A}_{p_{1} p_{2} p_{3} q}, \mathcal{P}(q), p_{1}\right) \\
& \sum_{\underline{q}^{1 / 4.4} \leq p_{1}<p_{2}<\underline{q}^{1 / 3.6}<\underline{q}^{1 / 3} \leq p_{3}<\underline{q}^{1 / 2.4}} S\left(\mathcal{A}_{p_{1} p_{2} p_{3} q}, \mathcal{P}(q), p_{1}\right) \\
& -\sum_{\underline{q}^{1 / 3} \leq p<\underline{q}^{1 / 2.2}} S\left(\mathcal{A}_{p q}, \mathcal{P}(q), p\right) \\
& \text { - } \sum_{\underline{q}^{1 / 2.4} \leq p<\underline{q}^{1 / 2.2}} S\left(\mathcal{A}_{p q}, \mathcal{P}(q), p\right) \\
& =U_{1}(q)+U_{2}(q)-V_{1}(q)-V_{2}(q)-V_{3}(q) \\
& -V_{4}(q)-V_{5}(q)-V_{6}(q) \text {, }
\end{aligned}
$$


$(3.10)$

$$
\begin{aligned}
& S_{q}^{3}= \frac{1}{2} \sum_{\underline{q}^{1 / 4.5} \leq p_{1}<p_{2}<\underline{q}^{1 / 4.4}} S\left(\mathcal{A}_{p_{1} p_{2} q}, \mathcal{P}(q), p_{1}\right) \\
&+\frac{1}{2} \sum_{\underline{q}^{1 / 4.5} \leq p_{1}<\underline{q}^{1 / 4.4} \leq p_{2}<\underline{q}^{1 / 2.2}} S\left(\mathcal{A}_{p_{1} p_{2} q}, \mathcal{P}(q), p_{1}\right) \\
&-\frac{1}{2} \sum_{\underline{q}^{1 / 4.5} \leq p<\underline{q}^{1 / 4.4}} S\left(\mathcal{A}_{p q}, \mathcal{P}(q), p\right) \\
&-\frac{1}{2} \sum_{\underline{q}^{1 / 4.5} \leq p_{1}<p_{2}<\underline{q}^{1 / 4.4} \leq p_{3}<\underline{q}^{1 / 2.2}} S\left(\mathcal{A}_{p_{1} p_{2} p_{3} q}, \mathcal{P}(q), p_{1}\right) \\
&-\frac{1}{2} S\left(\mathcal{A}_{p_{1} p_{2} p_{3} q}, \mathcal{P}(q), p_{1}\right) \\
&-\sum_{\underline{q}^{1 / 4.5} \leq p_{1}<p_{2}<p_{3}<\underline{q}^{1 / 4.4}} S\left(\mathcal{A}_{p_{1} p_{2} p_{3} q}, \mathcal{P}(q), p_{1}\right) \\
&-\underline{q}^{1 / 4.5} \leq p_{1}<\underline{q}^{1 / 4.4} \leq p_{2}<\underline{q}^{1 / 3.6}<\underline{q}^{1 / 3} \leq p_{3}<\underline{q}^{1 / 2.4} \\
& \sum_{q^{1 / 4.5} \leq p_{1}<\underline{q}^{1 / 4.4} \leq p_{2}<p_{3}<\underline{q}^{1 / 3}} S\left(\mathcal{A}_{p_{1} p_{2} p_{3} q}, \mathcal{P}(q), p_{1}\right) .
\end{aligned}
$$

We shall denote $O\left(\frac{x}{q \log ^{5 k+100} x}\right)$ by $E(q)$ below. By Buchstab's identity we have

$$
\begin{aligned}
V_{1}(q)= & \sum_{\underline{q}^{1 / 4.4} \leq p<\underline{q}^{1 / 2.2}} S\left(\mathcal{A}_{p q}, \mathcal{P}(q), \underline{q}^{1 / 2.2}\right) \\
& +\sum_{\underline{q}^{1 / 4.4} \leq p_{1} \leq p_{2}<\underline{q}^{1 / 2.2}} S\left(\mathcal{A}_{p_{1} p_{2} q}, \mathcal{P}\left(p_{1} q\right), p_{2}\right) \\
\geq & \sum_{\underline{q}^{1 / 4.4} \leq p_{1}<p_{2}<\underline{q}^{1 / 2.2}} S\left(\mathcal{A}_{p_{1} p_{2} q}, \mathcal{P}\left(p_{1} q\right), p_{2}\right),
\end{aligned}
$$

so that

$$
\begin{aligned}
U_{1}(q)-V_{1}(q) & \leq \sum_{\underline{q}^{1 / 4.4} \leq p_{1} \leq p_{2}<p_{3}<\underline{q}^{1 / 2.2}} S\left(\mathcal{A}_{p_{1} p_{2} p_{3} q}, \mathcal{P}\left(p_{1} q\right), p_{2}\right) \\
& \leq \sum_{\substack{\underline{q}^{1 / 4.4} \leq p_{1}<p_{2}<p_{3}<\underline{q}^{1 / 2.2}}} S\left(\mathcal{A}_{p_{1} p_{2} p_{3} q}, \mathcal{P}\left(p_{1} q\right), p_{2}\right)+E(q) \\
& =U_{3}(q)+E(q) .
\end{aligned}
$$

In a similar way, we have

$$
U_{2}(q)-V_{2}(q) \leq \sum_{\underline{q}^{1 / 3.6} \leq p_{1}<p_{2}<p_{3}<\underline{q}^{1 / 2.4}} S\left(\mathcal{A}_{p_{1} p_{2} p_{3} q}, \mathcal{P}\left(p_{1} q\right), p_{2}\right)
$$




$$
\begin{aligned}
& -\sum_{\underline{q}^{1 / 3.6} \leq p_{1}<\underline{q}^{1 / 2.4} \leq p_{2}<\underline{q}^{1 / 2.2}} S\left(\mathcal{A}_{p_{1} p_{2} q}, \mathcal{P}\left(p_{1} q\right), p_{2}\right) \\
& -\sum_{\underline{q}^{1 / 2.4} \leq p<\underline{q}^{1 / 2.2}} S\left(\mathcal{A}_{p q}, \mathcal{P}(q), p\right)+E(q) \\
& =W_{1}(q)-V_{7}(q)-V_{6}(q)+E(q), \\
& U_{3}(q)-V_{3}(q) \\
& \leq \sum_{\underline{q}^{1 / 4.4} \leq p_{1}<p_{2}<\underline{q}^{1 / 3} \leq p_{3}<\underline{q}^{1 / 2.2}} S\left(\mathcal{A}_{p_{1} p_{2} p_{3} q}, \mathcal{P}\left(p_{1} q\right), p_{2}\right) \\
& +\sum_{\underline{q}^{1 / 4.4} \leq p_{1}<\underline{q}^{1 / 3} \leq p_{2}<p_{3}<\underline{1}^{1 / 2.2}} S\left(\mathcal{A}_{p_{1} p_{2} p_{3} q}, \mathcal{P}\left(p_{1} q\right), p_{2}\right) \\
& +\sum_{\underline{q}^{1 / 3} \leq p_{1}<p_{2}<p_{3}<\underline{q}^{1 / 2.2}} S\left(\mathcal{A}_{p_{1} p_{2} p_{3} q}, \mathcal{P}\left(p_{1} q\right), p_{2}\right)+E(q) \\
& =U_{4}(q)+U_{5}(q)+U_{6}(q)+E(q), \\
& \text { (3.14) } \quad U_{4}(q)-V_{4}(q) \\
& \begin{aligned}
\leq & \sum_{\underline{q}^{1 / 4.4} \leq p_{1}<p_{2}<\underline{q}^{1 / 3.6}<\underline{q}^{1 / 2.4} \leq p_{3}<\underline{q}^{1 / 2.2}} S\left(\mathcal{A}_{p_{1} p_{2} p_{3} q}, \mathcal{P}\left(p_{1} q\right), p_{2}\right) \\
& +\sum_{\underline{q}^{1 / 4.4} \leq p_{1}<\underline{q}^{1 / 3.6} \leq p_{2}<\underline{q}^{1 / 3} \leq p_{3}<\underline{q}^{1 / 2.2}} S\left(\mathcal{A}_{p_{1} p_{2} p_{3} q}, \mathcal{P}\left(p_{1} q\right), p_{2}\right) \\
& +\sum_{\underline{q}^{1 / 3.6} \leq p_{1}<p_{2}<\underline{q}^{1 / 3} \leq p_{3}<\underline{q}^{1 / 2.2}} S\left(\mathcal{A}_{p_{1} p_{2} p_{3} q}, \mathcal{P}\left(p_{1} q\right), p_{2}\right)+E(q) \\
= & W_{2}(q)+W_{3}(q)+W_{4}(q)+E(q),
\end{aligned} \\
& \leq \sum_{\underline{q}^{1 / 4.4} \leq p_{1}<\underline{q}^{1 / 3.6}<\underline{q}^{1 / 3} \leq p_{2}<p_{3}<\underline{q}^{1 / 2.2}} S\left(\mathcal{A}_{p_{1} p_{2} p_{3} q}, \mathcal{P}\left(p_{1} q\right), p_{2}\right) \\
& +\sum_{\underline{q}^{1 / 3.6} \leq p_{1}<\underline{q}^{1 / 3} \leq p_{2}<p_{3}<\underline{q}^{1 / 2.4}} S\left(\mathcal{A}_{p_{1} p_{2} p_{3} q}, \mathcal{P}\left(p_{1} q\right), p_{2}\right) \\
& +\sum_{\underline{q}^{1 / 3.6} \leq p_{1}<\underline{q}^{1 / 3} \leq p_{2}<\underline{q}^{1 / 2.4} \leq p_{3}<\underline{q}^{1 / 2.2}} S\left(\mathcal{A}_{p_{1} p_{2} p_{3} q}, \mathcal{P}\left(p_{1} q\right), p_{2}\right) \\
& +\sum_{\underline{q}^{1 / 3.6} \leq p_{1}<\underline{q}^{1 / 3}<\underline{1}^{1 / 2.4} \leq p_{2}<p_{3}<p_{4}<\underline{q}^{1 / 2.2}} S\left(\mathcal{A}_{p_{1} p_{2} p_{3} q}, \mathcal{P}\left(p_{1} p_{2} q\right), p_{3}\right)
\end{aligned}
$$

(3.15) $\quad U_{5}(q)-V_{7}(q)$ 


$$
\begin{aligned}
& -\sum_{\underline{q}^{1 / 3} \leq p_{1}<\underline{q}^{1 / 2.4} \leq p_{2}<\underline{q}^{1 / 2.2}} S\left(\mathcal{A}_{p_{1} p_{2} q}, \mathcal{P}\left(p_{1} q\right), p_{2}\right)+E(q) \\
& =W_{5}(q)+W_{6}(q)+W_{7}(q)+W_{8}(q)-V_{8}(q)+E(q) \text {, } \\
& U_{6}(q)-V_{5}(q) \\
& \leq \sum_{\underline{q}^{1 / 3} \leq p_{1}<p_{2}<p_{3}<p_{4}<\underline{q}^{1 / 2.2}} S\left(\mathcal{A}_{p_{1} p_{2} p_{3} p_{4} q}, \mathcal{P}\left(p_{1} p_{2} q\right), p_{3}\right)+E(q) \\
& =U_{7}(q)+E(q), \\
& \text { (3.17) } \quad U_{7}(q)-2 V_{6}(q)-V_{8}(q) \\
& \leq \sum_{\underline{q}^{1 / 3} \leq p_{1}<p_{2}<p_{3}<p_{4}<\underline{q}^{1 / 2.4}} S\left(\mathcal{A}_{p_{1} p_{2} p_{3} p_{4} q}, \mathcal{P}\left(p_{1} p_{2} q\right), p_{3}\right) \\
& +\sum_{\underline{q}^{1 / 3} \leq p_{1}<p_{2}<p_{3}<\underline{q}^{1 / 2.4} \leq p_{4}<\underline{q}^{1 / 2.2}} S\left(\mathcal{A}_{p_{1} p_{2} p_{3} p_{4} q}, \mathcal{P}\left(p_{1} p_{2} q\right), p_{3}\right) \\
& +\sum_{\underline{q}^{1 / 3} \leq p_{1}<p_{2}<\underline{q}^{1 / 2.4} \leq p_{3}<p_{4}<\underline{q}^{1 / 2.2}} S\left(\mathcal{A}_{p_{1} p_{2} p_{3} p_{4} q}, \mathcal{P}\left(p_{1} p_{2} q\right), p_{3}\right) \\
& +\sum_{\underline{q}^{1 / 3} \leq p_{1}<\underline{q}^{1 / 2.4} \leq p_{2}<p_{3}<p_{4}<p_{5}<\underline{q}^{1 / 2.2}} S\left(\mathcal{A}_{p_{1} p_{2} p_{3} p_{4} q}, \mathcal{P}\left(p_{1} p_{2} p_{3} q\right), p_{4}\right) \\
& +\sum_{\Delta} S\left(\mathcal{A}_{p_{1} p_{2} p_{3} p_{4} p_{5} p_{6} q}, \mathcal{P}\left(p_{1} p_{2} p_{3} p_{4} q\right), p_{5}\right)+E(q) \\
& =\sum_{j=9}^{13} W_{j}(q)+E(q),
\end{aligned}
$$

where $\sum_{\Delta}$ means

$$
\sum_{\underline{q}^{1 / 2.4} \leq p_{1}<p_{2}<p_{3}<p_{4}<p_{5}<p_{6}<\underline{q}^{1 / 2.2}} .
$$

By (3.9), (3.11)-(3.17), we get

$$
S_{q}^{2} \leq \sum_{j=1}^{13} W_{j}(q)+E(q)
$$

Similarly, we have

$$
\begin{aligned}
& S_{q}^{3} \leq \frac{1}{2} \sum_{\underline{q}^{1 / 4.5} \leq p_{1}<\underline{q}^{1 / 4.4}<\underline{q}^{1 / 3} \leq p_{2}<p_{3}<\underline{q}^{1 / 2.2}} S\left(\mathcal{A}_{p_{1} p_{2} p_{3} q}, \mathcal{P}\left(p_{1} q\right), p_{2}\right) \\
& +\frac{1}{2} \sum_{\underline{q}^{1 / 4.5} \leq p_{1}<\underline{q}^{1 / 4.4} \leq p_{2}<\underline{q}^{1 / 3.6}<\underline{q}^{1 / 2.4} \leq p_{3}<\underline{q}^{1 / 2.2}} S\left(\mathcal{A}_{p_{1} p_{2} p_{3} q}, \mathcal{P}\left(p_{1} q\right), p_{2}\right)
\end{aligned}
$$




$$
\begin{aligned}
& +\frac{1}{2} \sum_{\underline{q}^{1 / 4.5} \leq p_{1}<\underline{q}^{1 / 4.4}<\underline{q}^{1 / 3.6} \leq p_{2}<\underline{q}^{1 / 3} \leq p_{3}<\underline{q}^{1 / 2.2}} S\left(\mathcal{A}_{p_{1} p_{2} p_{3} q}, \mathcal{P}\left(p_{1} q\right), p_{2}\right)+E(q) \\
= & \frac{1}{2}\left(W_{14}(q)+W_{15}(q)+W_{16}(q)\right)+E(q) .
\end{aligned}
$$

By Lemma 6, Lemma 5 and some routine arguments we get

$$
\begin{aligned}
& \sum \chi(q) W_{1}(q) \\
& \prec \frac{3.5 C}{\log x} \sum \chi(q) \sum_{\underline{q}^{1 / 3.6} \leq p_{1}<p_{2}<p_{3}<\underline{q}^{1 / 2.4}} \sum_{\substack{n \leq \frac{x}{p_{1} p_{2} p_{3}} \\
\left(n, Q\left(p_{2}\right)\right)=1}} 1 \\
& \prec \frac{3.5 C}{1.763 \log x} \sum \chi(q) \sum_{\underline{q}^{1 / 3.6} \leq p_{1}<p_{2}<p_{3}<\underline{q}^{1 / 2.4}} \frac{x}{p_{1} p_{2} p_{3} q \log p_{2}} \\
& \prec \frac{3.5 C x}{1.763 \log x} \sum \frac{\chi(q)}{\varphi(q)} \int_{\underline{q}^{1 / 3.6}} \frac{d r}{r \log r} \int_{r} \frac{d s}{s \log ^{2} s} \int_{s}^{\underline{q}^{1 / 2.4}} \frac{d t}{t \log t} \\
& \prec \frac{7}{4 \cdot 1.763}\left(6 \log \frac{3.6}{2.4}-2.4\right) \Xi(\chi ; x),
\end{aligned}
$$

where $A \prec B$ means $A \leq(1+o(1)) B$. Similarly, we have

(3.28) $\sum \chi(q) W_{9}(q) \prec \frac{7}{8}\left(2.4 \log ^{2} \frac{3}{2.4}+15.6 \log \frac{3}{2.4}-3.6\right) \Xi(\chi ; x)$,

(3.29) $\sum \chi(q) W_{10}(q) \prec \frac{7}{8}\left(1.2-4.8 \log \frac{3}{2.4}-2.4 \log ^{2} \frac{3}{2.4}\right) \log \frac{2.4}{2.2} \Xi(\chi ; x)$, 
(3.30)

$$
\begin{aligned}
& \text { (3.30) } \sum \chi(q) W_{11}(q) \prec \frac{7}{8}\left(2.4 \log \frac{2.4}{2.2}-0.2\right) \log ^{2} \frac{3}{2.4} \Xi(\chi ; x), \\
& \text { (3.31) } \sum \chi(q) W_{12}(q) \prec \frac{7}{8}\left(2.2 \log ^{2} \frac{2.4}{2.2}+13.6 \log \frac{2.4}{2.2}-1.2\right) \log \frac{3}{2.4} \Xi(\chi ; x), \\
& \text { (3.32) } \sum \chi(q) W_{13}(q) \prec \frac{7}{4 \cdot 6 !} \log ^{5} \frac{2.4}{2.2} \Xi(\chi ; x), \\
& \text { (3.33) } \sum \chi(q) W_{14}(q) \prec \frac{7}{4 \cdot 1.763}\left(3 \log \frac{3}{2.2}-0.8\right) \log \frac{4.5}{4.4} \Xi(\chi ; x), \\
& \text { (3.34) } \sum \chi(q) W_{15}(q) \prec \frac{7}{4 \cdot 1.763} \cdot 0.8 \log \frac{4.5}{4.4} \log \frac{2.4}{2.2} \Xi(\chi ; x), \\
& \text { (3.35) } \sum \chi(q) W_{16}(q) \prec \frac{7}{4 \cdot 1.763} \cdot 0.6 \log \frac{4.5}{4.4} \log \frac{3}{2.2} \Xi(\chi ; x),
\end{aligned}
$$

where we have used the prime number theorem and the formulae

$$
\begin{aligned}
\int_{y^{1 / \alpha}}^{y^{1 / \beta}} \frac{d r}{r \log ^{2} r} \int_{r}^{y^{1 / \beta}} \frac{d s}{s \log s} & =\frac{\alpha \log \frac{\alpha}{\beta}+\beta-\alpha}{\log y} \\
\int_{y^{1 / \alpha}}^{1 / \beta} \frac{d r}{r \log r} \int_{r}^{y^{1 / \beta}} \frac{d s}{s \log ^{2} s} & =\frac{\alpha-\beta-\beta \log \frac{\alpha}{\beta}}{\log y} \\
\int_{y^{1 / \alpha}}^{y^{1 / \beta}} \frac{d r}{r \log r} \int_{r}^{y^{1 / \beta}} \frac{d s}{s \log s} \int_{s}^{y^{1 / \beta}} \frac{d t}{t \log ^{2} t} & =\frac{\alpha-\beta-\beta \log \frac{\alpha}{\beta}-\frac{\beta}{2} \log ^{2} \frac{\alpha}{\beta}}{\log y}
\end{aligned}
$$$$
\int_{y^{1 / \alpha}}^{y^{1 / \beta}} \frac{d r}{r \log r} \int_{r}^{y^{1 / \beta}} \frac{d s}{s \log ^{2} s} \int_{s}^{y^{1 / \beta}} \frac{d t}{t \log t}=\frac{(\alpha+\beta) \log \frac{\alpha}{\beta}+2(\beta-\alpha)}{\log y},
$$

$$
\begin{gathered}
\int_{y^{1 / \alpha}}^{y^{1 / \beta}} \frac{d r}{r \log r} \int_{r}^{y^{1 / \beta}} \frac{d s}{s \log s} \int_{s}^{y^{1 / \beta}} \frac{d t}{t \log ^{2} t} \int_{w}^{y^{1 / \beta}} \frac{d w}{w \log w} \\
=\frac{\frac{\beta}{2} \log ^{2} \frac{\alpha}{\beta}+(\alpha+2 \beta) \log \frac{\alpha}{\beta}+3(\beta-\alpha)}{\log y}
\end{gathered}
$$

By (3.18)-(3.35) we get

$$
\begin{aligned}
& \sum \chi(q) S_{q}^{2} \leq \sum \chi(q) \sum_{j=1}^{13}\left(W_{j}(q)+E(q)\right)<0.14228 \Xi(\chi ; x) \\
& \sum \chi(q) S_{q}^{3} \leq \frac{1}{2} \sum \chi(q) \sum_{j=14}^{16}\left(W_{j}(q)+E(q)\right)<0.00431 \Xi(\chi ; x) .
\end{aligned}
$$


By the definition of $H(s), G(s)$, Lemma 3 and some routine arguments we get

$$
\begin{aligned}
& \sum \chi(q) S\left(\mathcal{A}_{q}, \mathcal{P}(q), \underline{q}^{1 / v}\right) \\
& \leq\left(1+\int_{2}^{v-1} \frac{\log (t-1)}{t} d t-H(v)\right) \Xi(\chi ; x), \\
& \sum \chi(q) \sum_{q^{1 / w} \leq p<\underline{q}^{1 / u}} S\left(\mathcal{A}_{p q}, \mathcal{P}(p q), \underline{q}^{1 / v}\right) \\
& \geq\left(\int_{u-1}^{w-1} \frac{\log \left(v-1-\frac{v}{t+1}\right)+G\left(v-\frac{v}{t+1}\right)}{t} d t-o(1)\right) \Xi(\chi ; x),
\end{aligned}
$$

where $3<u<w<v<5$, and

$$
\begin{aligned}
\sum \chi(q) & \sum_{\underline{q}^{1 / 4.5} \leq p_{1}<p_{2}<\underline{q}^{1 / 4.4}} S\left(\mathcal{A}_{p_{1} p_{2} q}, \mathcal{P}(q), \underline{q}^{1 / 4.5}\right) \\
\leq & \left(\int_{1 / 4.5}^{1 / 4.4} \int_{t_{1}}^{1 / 4.4} \frac{1-H(2.5)}{t_{1} t_{2}\left(1-t_{1}-t_{2}\right)} d t_{1} d t_{2}+o(1)\right) \Xi(\chi ; x),
\end{aligned}
$$

$$
\begin{aligned}
\sum \chi(q) & \sum_{\substack{\underline{q}^{1 / 4.5} \leq p_{1}<p_{2}<\underline{q}^{1 / 4.4} \\
\leq}\left(\mathcal{A}_{p_{1} p_{2} q}, \mathcal{P}(q), p_{1}\right)} \\
\leq & \left(\int_{1 / 4.5}^{1 / 4.4} \int_{t_{1}}^{1 / 4.4} \frac{1-H(2.5)}{t_{1} t_{2}\left(1-t_{1}-t_{2}\right)} d t_{1} d t_{2}+o(1)\right) \Xi(\chi ; x),
\end{aligned}
$$

$$
\begin{aligned}
\sum \chi(q) & \sum_{\underline{q}^{1 / 4.5} \leq p_{1}<\underline{q}^{1 / 4.4}<\underline{1}^{1 / 3} \leq p_{2}<\underline{q}^{1 / 2.4}} S\left(\mathcal{A}_{p_{1} p_{2} q}, \mathcal{P}(q), p_{1}\right) \\
\leq & \left(\int_{1 / 4.5}^{1 / 4.4} \int_{1 / 3}^{1 / 2.4} \frac{1-H(2.2)}{t_{1} t_{2}\left(1-t_{1}-t_{2}\right)} d t_{1} d t_{2}+o(1)\right) \Xi(\chi ; x),
\end{aligned}
$$

$$
\begin{aligned}
& \sum \chi(q) \sum_{\underline{q}^{1 / 4.4} \leq p_{1}<\underline{q}^{1 / 3.6}<\underline{q}^{1 / 3} \leq p_{2}<\underline{q}^{1 / 2.4}} S\left(\mathcal{A}_{p_{1} p_{2} q}, \mathcal{P}(q), \underline{q}^{1 / 4.5}\right) \\
& \leq\left(\int_{1 / 4.4}^{1 / 3.6} \int_{1 / 3}^{1 / 2.4} \frac{1-H(2.2)}{t_{1} t_{2}\left(1-t_{1}-t_{2}\right)} d t_{1} d t_{2}+o(1)\right) \Xi(\chi ; x),
\end{aligned}
$$

$$
\sum \chi(q) \sum_{\underline{q}^{1 / 4.4} \leq p_{1}<p_{2}<\underline{q}^{1 / 3}} S\left(\mathcal{A}_{p_{1} p_{2} q}, \mathcal{P}(q), \underline{q}^{1 / 4.5}\right)
$$


(3.46)

$$
\leq\left(\int_{1 / 4.4}^{1 / 3} \int_{t_{1}}^{1 / 3} \frac{1-H\left(4.5\left(1-t_{1}-t_{2}\right)\right)}{t_{1} t_{2}\left(1-t_{1}-t_{2}\right)} d t_{1} d t_{2}+o(1)\right) \Xi(\chi ; x),
$$

$$
\begin{aligned}
& \sum \chi(q) \sum_{\underline{q}^{1 / 4.5} \leq p_{1}<\underline{q}^{1 / 4.4} \leq p_{2}<\underline{q}^{1 / 2.2}} S\left(\mathcal{A}_{p_{1} p_{2} q}, \mathcal{P}(q), \underline{q}^{1 / 4.5}\right) \\
& \leq\left(\int_{1 / 4.5}^{1 / 4.4} \int_{1 / 4.4}^{1 / 2.2} \frac{1-H\left(4.5\left(1-t_{1}-t_{2}\right)\right)}{t_{1} t_{2}\left(1-t_{1}-t_{2}\right)} d t_{1} d t_{2}+o(1)\right) \Xi(\chi ; x),
\end{aligned}
$$

$$
\begin{aligned}
\sum \chi(q) & \sum_{\underline{q}^{1 / 4.4} \leq p_{1}<p_{2}<\underline{q}^{1 / 3.6}} S\left(\mathcal{A}_{p_{1} p_{2} q}, \mathcal{P}(q), \underline{q}^{1 / 4.5}\right) \\
\leq & \left(\int_{1 / 4.4}^{1 / 3.6} \int_{t_{1}}^{1 / 3.6} \frac{1-H\left(\frac{1-t_{1}-t_{2}}{t_{1}}\right)}{t_{1} t_{2}\left(1-t_{1}-t_{2}\right)} d t_{1} d t_{2}+o(1)\right) \Xi(\chi ; x),
\end{aligned}
$$

$$
\begin{aligned}
& \sum \chi(q) \sum_{\underline{q}^{1 / 4.4} \leq p_{1}<\underline{q}^{1 / 3.6} \leq p_{2}<\underline{q}^{1 / 3}} S\left(\mathcal{A}_{p_{1} p_{2} q}, \mathcal{P}(q), \underline{q}^{1 / 4.5}\right) \\
& \leq\left(\int_{1 / 4.4}^{1 / 3.6} \int_{1 / 3.6}^{1 / 3} \frac{1-H\left(4.5\left(1-t_{1}-t_{2}\right)\right)}{t_{1} t_{2}\left(1-t_{1}-t_{2}\right)} d t_{1} d t_{2}+o(1)\right) \Xi(\chi ; x) .
\end{aligned}
$$

By (3.38)-(3.47) and (3.8) we get

$$
S_{q}^{1} \leq\left(N_{1}-N_{2}\right) \Xi(\chi ; x),
$$

where

$$
\begin{aligned}
\text { (3.49) } N_{1}= & +\int_{2}^{3.5} \frac{\log (t-1)}{t} d t+\int_{2}^{3.4} \frac{\log (t-1)}{t} d t+2 \int_{2}^{2.6} \frac{\log (t-1)}{t} d t \\
& -\frac{1}{2} \int_{1.2}^{3.5} \frac{\log \left(3.5-\frac{4.5}{t+1}\right)}{t} d t-\frac{1}{2} \int_{1.2}^{3.4} \frac{\log \left(3.4-\frac{4.4}{t+1}\right)}{t} d t \\
& -\int_{1.4}^{2.6} \frac{\log \left(2.6-\frac{3.6}{t+1}\right)}{t} d t \\
\leq & 5-0.244058, \\
\text { (3.50) } \quad N_{2}= & 2 H(4.5)+2 H(4.4)+H(3.6)+\int_{1.2}^{3.5} \frac{G\left(4.5-\frac{4.5}{t+1}\right)}{t} d t
\end{aligned}
$$$$
+\frac{1}{2} \int_{3.4}^{3.5} \frac{G\left(4.5-\frac{4.5}{t+1}\right)}{t} d t+2 \int_{2}^{3.4} \frac{G\left(4.4-\frac{4.4}{t+1}\right)}{t} d t
$$ 


$$
\begin{aligned}
& +\int_{1.4}^{2} \frac{G\left(4.5-\frac{4.5}{t+1}\right)}{t} d t+\int_{1 / 4.5}^{1 / 4.4} \int_{t_{1}}^{1 / 4.4} \frac{H(2.5)}{t_{1} t_{2}\left(1-t_{1}-t_{2}\right)} d t_{1} d t_{2} \\
& +\frac{1}{2} \int_{1 / 4.5}^{1 / 4.4} \int_{1 / 4.4}^{1 / 2.2} \frac{H\left(4.5\left(1-t_{1}-t_{2}\right)\right)}{t_{1} t_{2}\left(1-t_{1}-t_{2}\right)} d t_{1} d t_{2} \\
& +\int_{1 / 4.4}^{1 / 3} \int_{t_{1}}^{1 / 3} \frac{H\left(4.5\left(1-t_{1}-t_{2}\right)\right)}{t_{1} t_{2}\left(1-t_{1}-t_{2}\right)} d t_{1} d t_{2} \\
& +\int_{1 / 3.6}^{1 / 3} \int_{1 / 3.4}^{1 / 3.6} \frac{H\left(4.5\left(1-t_{1}-t_{2}\right)\right)}{t_{1} t_{2}\left(1-t_{1}-t_{2}\right)} d t_{1} d t_{2} \\
& +\int_{1 / 4.4}^{1 / 3.6} \frac{H\left(\frac{1-t_{1}-t_{2}}{t_{1}}\right)}{t_{1}} d t_{1} d t_{2} \\
& +\int_{1 / 3.6} \int_{1 / 2.4} \frac{H(2.2)}{t_{1} t_{2}\left(1-t_{1}-t_{2}\right)} d t_{1} d t_{2} \\
& +\int_{1 / 4.4} \int_{1 / 2.4} \frac{H(2.2)}{\left.t_{1} t_{1}-t_{2}\right)} d t_{1} d t_{2} .
\end{aligned}
$$

By (2.6) we have

(3.51) $2 H(4.5)+2 H(4.4)+H(3.6)$

$$
\begin{aligned}
\geq & 1.02288 G(4)+0.03245 H(2.2)+0.0099 H(2.3)+0.01078 H(2.4) \\
& +0.01272 H(2.5)+0.01668 H(2.6)+0.02130 H(2.7) \\
& +0.02544 H(2.8)+0.02916 H(2.9) \\
& +0.03252 H(3)-\varepsilon .
\end{aligned}
$$

By (2.7) we have

$$
\begin{aligned}
\left(\int_{1.2}^{3.5}+\frac{1}{2} \int_{3.4}^{3.5}\right. & \left.+\int_{1.4}^{2}\right) \frac{G\left(4.5-\frac{4.5}{t+1}\right)}{t} d t+2 \int_{2}^{3.4} \frac{G\left(4.4-\frac{4.4}{t+1}\right)}{t} d t \\
\geq & 2.50285 G(4)+0.23871 H(2.2)+0.08093 H(2.3) \\
& +0.09264 H(2.4)+0.09912 H(2.5)+0.09816 H(2.6) \\
& +0.09444 H(2.7)+0.09102 H(2.8)+0.08782 H(2.9) \\
& +0.08485 H(3)-\varepsilon
\end{aligned}
$$


By Proposition 1(1) we have

$$
\begin{aligned}
\int_{1 / 4.5}^{1 / 4.4} \int_{t_{1}}^{1 / 4.4} \frac{H(2.5)}{t_{1} t_{2}\left(1-t_{1}-t_{2}\right)} d t_{1} d t_{2} & \\
& +\frac{1}{2} \int_{1 / 4.5}^{1 / 4.4} \int_{1 / 4.4}^{1 / 2.2} \frac{H\left(4.5\left(1-t_{1}-t_{2}\right)\right)}{t_{1} t_{2}\left(1-t_{1}-t_{2}\right)} d t_{1} d t_{2} \\
& +\int_{1 / 4.4}^{1 / 3} \int_{t_{1}}^{1 / 3} \frac{H\left(4.5\left(1-t_{1}-t_{2}\right)\right)}{t_{1} t_{2}\left(1-t_{1}-t_{2}\right)} d t_{1} d t_{2} \\
& +\int_{1 / 4.6}^{1 / 3} \int_{1 / 2.4}^{1 / 3} \frac{H(2.2)}{t_{1} t_{2}\left(1-t_{1}-t_{2}\right)} d t_{1} d t_{2} \\
& +\int_{1 / 4.5}^{1 / 4.4} \int_{1 / 2}^{1 / 2.4} \frac{H(2.2)}{t_{1} t_{2}\left(1-t_{1}-t_{2}\right)} d t_{1} d t_{2} \\
& +\int_{1 / 4.4}^{1 / 3.6} \int_{1 / 3.6}^{1 / 3} \frac{H\left(4.5\left(1-t_{1}-t_{2}\right)\right)}{t_{1} t_{2}\left(1-t_{1}-t_{2}\right)} d t_{1} d t_{2} \\
& +\int_{1 / 4.4}^{1 / 3.6} \int_{t_{1}}^{1 / 3.6} \frac{H\left(\frac{1-t_{1}-t_{2}}{t_{1}}\right)}{t_{1} t_{2}\left(1-t_{1}-t_{2}\right)} d t_{1} d t_{2} \\
\geq & 0.39974 H(2.2)+0.04041 H(2.5) .
\end{aligned}
$$

By (3.50)-(3.53) we have

(3.54) $\quad N_{2} \geq 0.74978 H(2.2)+0.10644 H(2.3)+0.1203 H(2.4)$

$$
\begin{aligned}
& +0.1704 H(2.5)+0.13423 H(2.6)+0.13629 H(2.7) \\
& +0.13814 H(2.8)+0.13972 H(2.9)+0.1412 H(3)-\varepsilon,
\end{aligned}
$$

where Proposition 3 is used.

By (3.7), (3.36), (3.37), (3.48) and (3.49) we have

$(3.55) \quad 5 \sum \chi(q) S\left(\mathcal{A}_{q}, \mathcal{P}(q), \underline{q}^{1 / 2.2}\right) \leq\left(5-0.097465-N_{2}\right) \Xi(\chi ; x)$.

By (3.54), (3.55) and the definition of $H(s)$ we get

$$
\begin{aligned}
H(2.2) \geq & 0.019493+0.14995 H(2.2)+0.02129 H(2.3) \\
& +0.02406 H(2.4)+0.03408 H(2.5)+0.02684 H(2.6) \\
& +0.02725 H(2.7)+0.02762 H(2.8)+0.02794 H(2.9) \\
& +0.02824 H(3),
\end{aligned}
$$




$$
\begin{aligned}
H(2.2) \geq & 0.022931+0.02504 H(2.3)+0.02830 H(2.4) \\
& +0.04009 H(2.5)+0.03157 H(2.6)+0.03205 H(2.7) \\
& +0.03249 H(2.8)+0.03286 H(2.9)+0.03322 H(3) .
\end{aligned}
$$

By Lemmas 8, 9 and 11 and (3.56) we get

$$
\begin{aligned}
& H(2.2) \geq 0.02685+0.000073+0.00137 H(2.2), \\
& H(2.2) \geq 0.02695 .
\end{aligned}
$$

Proof of the Theorem. Let $\chi$ denote the characteristic function of the set $\{1\}$. By the definition of $H(s)$, Proposition $1(2)$ and (3.57) we have

$$
\begin{aligned}
\pi_{2}(x) & \leq S\left(\mathcal{A}, \mathcal{P}, \underline{1}^{1 / 2.2}\right)+O\left(\underline{1}^{1 / 2.2}\right) \\
& \leq 3.5(1+o(1)) \frac{C x}{\log ^{2} x}(1-H(2.2))+O\left(\underline{1}^{1 / 2.2}\right) \\
& <\frac{3.406 C x}{\log ^{2} x} .
\end{aligned}
$$

The proof of the Theorem is complete.

\section{References}

[1] E. Bombieri and H. Davenport, Small differences between prime numbers, Proc. Roy. Soc. Ser. A 239 (1966), 1-18.

[2] E. Bombieri, J. B. Friedlander and H. Iwaniec, Primes in arithmetic progressions to large moduli, Acta Math. 156 (1986), 203-252.

[3] V. Brun, Le crible d'Eratosthène et le théorème de Goldbach, C. R. Acad. Sci. Paris 168 (1919), 544-546.

[4] J. R. Chen, On the Goldbach's problem and the sieve method, Sci. Sin. 21 (1978), 701-739.

[5] - On the representation of a large even integer as the sum of a prime and the product of at most two primes, ibid. 16 (1973), 157-176.

[6] E. Fouvry, Autour du théorème de Bombieri-Vinogradov, Acta Math. 152 (1984), 219-244.

[7] E. Fouvry and F. Grupp, On the switching principle in sieve theory, J. Reine Angew. Math. 370 (1986), 101-126.

[8] E. Fouvry and H. Iwaniec, Primes in arithmetic progressions, Acta Arith. 42 (1983), 197-218.

[9] H. Halberstam and H. E. Richert, Sieve Methods, Academic Press, London, 1974.

[10] G. H. Hardy and J. E. Littlewood, Some problems of 'partitio numerorum', III: On the expression of a number as a sum of primes, Acta Math. 44 (1923), 1-70.

[11] L. K. Hua, A direct attempt to Goldbach problem, Acta Math. Sinica 5 (1989), 1-8.

[12] H. Iwaniec, A new form of the error term in the linear sieve, Acta Arith. 37 (1980), 307-320.

[13] C. D. Pan, A new application of the Yu. V. Linnik large sieve method, Chinese Math. Acta 5 (1964), 642-652.

[14] —, A new attempt on Goldbach conjecture, Chinese Ann. Math. 3 (1982), 555-560. 
[15] A. Selberg, On elementary methods in prime number-theory and their limitations, in: 11 Skand. Mat. Kongr., Trondheim, 1949, 13-22.

[16] Y. Wang, On the representation of large integer as a sum of a prime and an almost prime, Sci. Sin. 11 (1962), 1033-1054.

[17] J. Wu, Sur la suite des nombres premiers jumeaux, Acta Arith. 55 (1990), 365-394.

Yingchun Cai

Institute of Mathematics

Tongji University

Shanghai 200092, P.R. China

E-mail: yccai@mail.shu.edu.cn
Minggao Lu

Department of Mathematics

Shanghai University

Shanghai 200436, P.R. China

E-mail: lumg0202@online.sh.cn

Received on 29.7.2002

and in revised form on 12.11.2002 